\title{
Increased Human Pathogenic Potential of Escherichia coli from Polymicrobial Urinary Tract Infections in Comparison to Isolates from Monomicrobial Culture Samples
}

Gemma Croxall ${ }^{1}$, Vivienne Weston ${ }^{2}$, Susan Joseph ${ }^{1}$, Georgina Manning ${ }^{1}$, Phil Cheetham $^{1}$, Alan McNally ${ }^{1 *}$

1 Pathogen Research Group, Nottingham Trent University, Clifton Lane, Nottingham, NG11 8NS

${ }^{2}$ Nottingham University Hospitals, Nottingham, England.

"Corresponding author: Dr Alan McNally, Pathogen Research Group, School of Science and Technology, Nottingham Trent University, Clifton Lane, Nottingham NG11 8NS.

Tel: $0044+1158483324$

Email: alan.mcnally@ntu.ac.uk

Running title: Pathogens in mixed culture UTI samples 


\section{Abstract}

2 The current diagnostic standard procedure outlined by the HPA for urinary tract 3 infections (UTI) in clinical laboratories does not report bacteria isolated from

4 samples containing 3 or more different bacterial species. As a result many UTI go

5 unreported and untreated, particularly in elderly patients, where polymicrobial UTI

6 samples are especially prevalent. This study reports the presence of the major

7 uropathogenic species in mixed culture urine samples from elderly patients, and

8 of resistance to front line antibiotics, with potentially increased levels of

9 resistance to ciprofloxacin and trimethoprim. Most importantly, the study

10 highlights that $E$. coli present in polymicrobial UTI samples are statistically more

11 invasive $(P<0.001)$ in in vitro epithelial cell infection assays than those isolated

12 from monomicrobial culture samples. In summary this study suggests that the

13 current diagnostic standard procedure for polymicrobial UTI samples needs to be

14 re-assessed, and that E. coli present in polymicrobial UTI samples may pose an

15 increased risk to human health.

16

17 Key words: Urinary tract infection, polymicrobial, E. coli, antimicrobial, invasion 
20 The annual incidence of UTI in the elderly population ranges from $10 \%$ in the

21 community to as high as $30 \%$ of hospitalised patients (Cove-Smith \& Almond,

22 2007). Mortality rates in elderly patients from bacteraemia as a result of UTI can

23 be as high as 33\% (Tal et al., 2005). A study by Plowman et al. (2001) found that

24 over a 12-month period urinary tract infections had the highest incidence (35\%)

25 of all nosocomial infections in a district general hospital, and the majority of

26 patients were over 60 years of age. UTI are also the most common infection in

27 long term care facilities, where they account for $20-60 \%$ of all antibiotic

28 prescriptions (Nicolle, 2001a). This large-scale prescription of antibiotics may well

29 contribute to the levels of antibiotic resistance in urinary pathogens (Zhanel et al.,

30 2005). The main etiologic agent of UTI is well documented as E. coli (Nicolle et

31 al., 2001b, Farajnia et al., 2008, \& Kumazawa \& Matsumoto, 1997). The

32 establishment of urinary tract pathogens is thought to begin with the invasion of

33 the superficial bladder epithelium, where bacteria can form intracellular

34 communities and receive a level of protection against the host immune system

35 and antibiotic treatment (Anderson et al., 2003, Blango \& Mulvey, 2010).

36 The diagnosis of UTI is routinely performed in the clinical laboratory by

37 microbiological culture of a urine sample according to the national standard

38 method developed and approved by the Health protection Agency

39 (http://www.hpa-standardmethods.org.uk/documents/bsop/pdf/bsop41.pdf).

40 Infections caused by a single organism are usually treatable with an antibiotic

41 regimen advised on the basis of antibiotic susceptibility tests. However, it has

42 been estimated that $33 \%$ of urine cultures from elderly patients are polymicrobial 
43 (Cove-Smith \& Almond, 2007). Due to the difficulties of identifying the organisms

44 present in these cultures clinical microbiology laboratories do not report

45 organisms isolated from urine in mixed culture unless there is a significant count

46 of a predominant organism

47 As a result there is insufficient information on the bacteria that cause

48 polymicrobial UTI and the threat they pose to patient health. This study aimed to

49 isolate the organisms present in polymicrobial urinary tract infection samples

50 from a population of elderly patients, and compare their prevalence, phenotypic

51 activity and pathogenic potential to monomicrobial culture isolates from the same

52 population, with the aim of further understanding the potential threat posed by

53 bacteria present in polymicrobial urine samples from elderly patients, and

54 possibly reassessing the current diagnostic standard procedure.

56 Materials and Methods

\section{Sample collection}

59 Two hundred and fifty urine culture agar plates were collected from Nottingham

60 University Hospitals (NUH) between October 2008 and June 2009. Cultures were

61 collected anonymously (therefore no ethical approval or informed consent

62 required) from patients aged 70 and over and assigned to one of the following

63 categories; (1) non-catheterized male polymicrobial infection, (2) non-

64 catheterized female polymicrobial infection, (3) catheterized male polymicrobial

65 infection, (4) catheterized female polymicrobial infection, all of which contained 3

66 or more organisms with no predominant count of one species. A fifth group, 
67 monomicrobial infection (uncatheterized or catheterized, males or females), was

68 also included for use as a control group. Bacteria were isolated using standard

69 microbiological identification procedures. Identification of isolates to species level

70 was performed using the API identification systems, API 20E, API 20strep, API

7120 NE and API Staph (Biomerieux,).

\section{$72 \quad$ Antibiotic susceptibility testing}

73 A breakpoint method was employed to obtain antibiotic susceptibility profiles for

74 all E. coli, S. aureus, E. faecalis and P. mirabilis. The BSAC method for

75 antimicrobial susceptibility testing was followed to prepare standardised inocula

76 (Andrews, 2009). The antibiotic panel used was as follows; Gentamicin $(2 \mu \mathrm{g} / \mathrm{ml})$,

77 Cefotaxime $(1 \mu \mathrm{g} / \mathrm{ml})$, Ceftazidime $(1 \mu \mathrm{g} / \mathrm{ml})$, Meropenem $(2 \mu \mathrm{g} / \mathrm{ml})$, Piperacillin-

78 tazobactam $(16 \mu \mathrm{g} / \mathrm{ml})$, Co-amoxiclav $(32 \mu \mathrm{g} / \mathrm{ml})$, Trimethoprim $(2 \mu \mathrm{g} / \mathrm{ml})$,

79 Ciprofloxacin $(4 \mu \mathrm{g} / \mathrm{ml})$, Cephradine $(32 \mu \mathrm{g} / \mathrm{ml})$, Nitrofurantoin $(32 \mu \mathrm{g} / \mathrm{ml})$, and

80 Amoxicillin $(32 \mu \mathrm{g} / \mathrm{ml})$. All $P$. aeruginosa isolates were tested using the BSAC

81 disc diffusion method (Andrews, 2009) and an antibiotic panel specific to

82 Pseudomonas; Gentamicin $(10 \mu \mathrm{g} / \mathrm{ml})$, Piperacillin-tazobactam $(85 \mu \mathrm{g} / \mathrm{ml})$,

83 Ceftazidime $(30 \mu \mathrm{g} / \mathrm{ml})$, Meropenem $(10 \mu \mathrm{g} / \mathrm{ml})$ and Ciprofloxacin $(1 \mu \mathrm{g} / \mathrm{ml})$.

84 PCR detection of $\beta$-lactamase genes

85 All E. coli, Klebsiella, Proteus, Pseudomonas, Enterobacter and Citrobacter

86 isolates were tested for the presence of the $\beta$-lactamase genes, bla $a_{\mathrm{TEM}}, b / a_{\mathrm{SHV}}$,

87 bla $a_{\text {СтX-M }}$ and bla $a_{\text {OX }}$ using a previously described multiplex PCR (Fang et al.,

88 2008). GenElute extraction kits (SIGMA) were used to extract bacterial genomic

89 DNA. Reference strains containing known $\beta$-lactamase types (NCTC 13351 E. 
90 coli blateM-3, NCTC 13353 E. coli blaoXA, blaСtX-M-15, and blateM and NCTC 13368

91 Klebsiella pneumoniae bla $\mathrm{TEM}_{\mathrm{T}}$, blas $\mathrm{STV}_{-18}$ ) were included as controls.

\section{ESBL confirmation tests}

93 ESBL combination ID discs were used to confirm the extended spectrum activity

94 of selected $\beta$-lactamase positive strains. The BSAC method for antimicrobial

95 susceptibility testing (Andrews, 2009) was followed to prepare a standardised

96 inoculum, which was used to inoculate an Iso-Sensitest agar plate to produce a

97 lawn of growth. Two sets of combination discs were used to optimise ESBL

98 detection, Cefpodoxime/Cefpodoxime\&clavulanate and

99 Cefepime/Cefepime\&clavulanate. An increase in zone of inhibition diameter of

$100 \geq 5 \mathrm{~mm}$ of the cephalosporin/clavulanic acid disc compared to the cephalosporin

101 alone indicated the presence of an ESBL-producing organism. Reference

102 organisms E. coli NCTC 13351, E. coli NCTC 13352 and E. coli NCTC 13353

103 and E. coli NCTC 10418 (HPA culture collections) were used for control 104 purposes.

105 Detection of vancomycin resistant enterococci, MRSA and Panton106 Valentine Leukocidin producing S. aureus

107 A previously published protocol, (Jayaratne et al., 1999) was used to screen all

108 E. faecalis and E. faecium isolates for the presence of vanA and vanB genes.

109 GenElute extraction kits (SIGMA) were used to extract bacterial genomic DNA. E.

110 faecalis NCTC 12201 was used as a reference strain.

111 A real-time PCR protocol developed by Thomas et al, (2007) was used to detect

112 the presence of the mecA gene, which confers resistance to methicillin, in all

113 Staphylococcus aureus isolates. PCR was also performed for the detection of the 
114 PVL-encoding gene, lukF, using a previously published protocol (Ribeiro et al.,

115 2005). The BSAC disc diffusion protocol (Andrews, 2009) was followed to test all

116 S. aureus isolates for phenotypic resistance to cefoxitin, which is indicative of

117 methicillin resistance, and a selection of Enterococcus isolates for phenotypic

118 resistance to vancomycin.

\section{BOX-PCR}

120 To confirm the organisms isolated from the UTI cultures were not all related to a 121 single locally disseminated clone, BOX-PCR was performed using a protocol 122 adapted from that of Koeuth et al, (1995). Extraintestinal pathogenic Escherichia

123 coli (ExPEC) CFT073 was used as a reference strain. PCR products were

124 examined using bionumerics software (BioNumerics v.3.5, Applied Maths), using

125 CFT073 to monitor the repeatability of the experiment.

\section{Cell cultures}

127 T24 human epithelial cells (HPA cultures) were grown in McCoy's 5A modified 128 media (Sigma, UK) supplemented with 10\% fetal bovine serum (Sigma, UK) and $1290.75 \%$ L-glutamine (Sigma, UK). Cells were grown in an atmosphere with 5\% $130 \mathrm{CO}_{2}$ at $37^{\circ} \mathrm{C}$ and sub-cultured twice-weekly. Two days prior to cell infection 131 assays, the T24 cells were seeded into 24 -well plates

\section{Association and invasion assays}

133 All E. coli assays were performed in duplicate on different days, and in triplicate

134 wells in each assay. Bacteria were cultured overnight in LB broth, harvested by 135 centrifugation and re-suspended in supplemented tissue culture medium, which 136 was then adjusted to $2 \times 10^{7} \mathrm{cfu} / \mathrm{ml}$, giving an $\mathrm{MOI}$ of $1: 100$. For E. coli, all 129 137 polymicrobial ExPEC and 21 monomicrobial ExPEC isolated in this study were 
138 quantifiably assayed using classical gentamicin protection assays as described

139 previously (McNally et al., 2007), using cultured T24 bladder cells. The invasive

140 ExPEC type strain, CFT073 was used as a positive control strain in all assays

141 and E.coli DH5a was used as a negative control strain. The mean number of

142 invasive bacteria was determined by Miles \& Misra plate counts from triplicate

143 wells. Strains that showed more than a 10-fold increase in invasion compared to

144 CFT073 were classed as highly-invasive strains. Those that showed more than a

145 10-fold decrease in invasion compared to CFT073 were deemed to be strains of

146 limited invasive potential.

147 All E. faecalis, $P$. mirabilis, $P$. aeruginosa and $S$. aureus were subjected to a

148 semi-quantitative screen performed in triplicate on different days to assess levels

149 of invasion. Assays were completed using the above method, which was adapted

150 for 96-well plates as described by Javed et al, (2009).

151 Statistical analysis

$152 \mathrm{X}^{2}$ tests were performed to compare invasion and antimicrobial resistance

153 between the monomicrobial culture and mixed culture populations, and also

154 bacterial prevalence in different patient groups.

156 Results

157 Similar bacterial species are isolated from monomicrobial and

158 polymicrobial UTI samples

159 Urine culture plates were collected from 250 patients over a 9-month period from

160 Nottingham University Hospitals (NUH) and were taken from both hospitalised

161 and community patients, with the median age of patients being 83.5 years. Of the 
162200 polymicrobial cultures collected, 71 (36\%) contained 2 organisms, 90 (45\%)

163 contained 3 organisms, $36(18 \%)$ contained 4 organisms and $3(1 \%)$ contained 5

164 organisms. Eighty three patients (33\%) had previous history of UTI, 27 cases of

165 which were designated as 'mixed' by the NUH clinical laboratory. Of these 27

166 patients presenting with a polymicrobial UTI, 13 went on to have further

167 incidences of polymicrobial UTI after this study, 5 patients went on to have a UTI

168 sample with a confirmed organism in monomicrobial culture and 9 had no further 169 infections.

170 A total of 620 bacterial strains were isolated from the 250 urine cultures and 171 identified to species level using API identification systems (figure 1). The most

172 predominant organism in both catheterised and uncatheterised patients was $E$.

173 coli, which was also far more prevalent in female patient cultures $(83 \%)$ than

174 male cultures (46\%) $\mathrm{P}<0.001$. In male samples $E$. faecalis was equally as

175 ubiquitous as $E$. coli. These two organisms were also frequently associated in 176 mixed cultures (36\%). E. coli was isolated from $68 \%$ of polymicrobial cultures and $17748 \%$ of monomicrobial cultures, and E. faecalis was isolated from $55 \%$ of 178 polymicrobial cultures, but was significantly less frequently isolated from

179 monomicrobial cultures (8\%), $\mathrm{P}<0.001$. $P$. aeruginosa, $P$. mirabilis and $S$. aureus

180 were also frequently isolated from $23 \%, 25 \%$ and $10.5 \%$ of polymicrobial cultures 181 respectively.

182 BOX-PCR profiles provide evidence of a genetically diverse population

183 In order to ensure that the organisms isolated were not epidemic clones, clonal

184 relatedness of E. coli, E. faecalis, P. mirabilis, $P$. aeruginosa and $S$. aureus 185 isolates was determined by BOX-PCR. BOX-group numbers were allocated 
based upon a similarity threshold of $92 \%$ which was decided upon after reviewing

187 previous publications (Proudy et al., 2008, \& Yang et al., 2004). The E. coli

188 strains exhibited varying BOX-profiles (figure 2) and did not appear to belong to a

189 dominant epidemic clone. Nineteen distinct clonal groups of E. coli were

190 assigned, the largest of which encompassed 71 of the total 150 E. coli strains in

191 the collection. Eleven distinct BOX-groups of E. faecalis were detected, with the

192 majority of isolates belonging to 2 of the groups, group 1 containing 53 isolates

193 and group 3 containing 37 isolates. Eighteen of the $51 \mathrm{P}$. aeruginosa isolates

194 were deemed to belong to the same BOX-group, and other isolates were also

195 allocated to 11 other BOX-groups. P. mirabilis were allocated 5 BOX-groups in

196 total, with $88 \%$ of strains found to belong to a single BOX-group. S. aureus were

197 allocated to 7 BOX-groups with $50 \%$ of the total strains belonging to one group.

198 These results suggest that the isolates are not derived from a single epidemic

199 clone of each species, and are indeed individual strains which could reasonably

200 be expected to possess varying phenotypic and genotypic properties.

201 Antibiotic resistance is comparable between bacteria from mixed and

202 monomicrobial cultures

203 To determine the specific antibiotic resistances within the population of

204 polymicrobial UTI organisms, 394 isolates of the 5 most commonly isolated

205 species (E. coli, E. faecalis, P. aeruginosa, P. mirabilis and S. aureus) were

206 subjected to antibiotic susceptibility tests using an antibiotic panel presently used

207 in the NUH clinical laboratory (table 1). Results suggest that bacteria isolated

208 from mixed culture samples exhibit comparable levels of resistance to front line

209 antibiotics as that observed in isolates from monomicrobial culture samples, 
210 regardless of species. With respect to $E$. coli, the percentage of mixed culture

211 sample isolates exhibiting resistance to ciprofloxacin and trimethoprim was

212 higher than that observed in monomicrobial culture sample isolates, and also

213 higher than the levels observed routinely in clinical urinary tract infection isolates.

214 The differences observed in this study are not statistically significant but require

215 further specific investigation.

216 Increased detection of Extended Spectrum $\beta$-Lactamases in monomicrobial

217 culture isolates

218 Due to the increasing prevalence of extended-spectrum $\beta$-lactamases (ESBLs) in

219 clinical samples the UTI isolates were screened both for the possession of $\beta$ -

220 lactamase genes and also for any ESBL phenotypic activity. All E. coli,

221 Enterobacter, Citrobacter, Klebsiella, Proteus and Pseudomonas strains $(n=355)$

222 were screened for the presence of blatem, blasHv, blactX-м, and blaoxA genes

223 using a previously published protocol (Fang et al., 2008). Forty-nine percent of

224 strains were found to possess a $\beta$-lactamase gene, and one fifth of the strains

225 possessed multiple bla genes (table 2). Interestingly most of the strains

226 containing multiple $\beta$-lactamases originated from monomicrobial culture

227 infections $(P=0.009)$. Klebsiella species were found to possess the most $\beta$ -

228 lactamases (93\% positive), $61 \%$ of $E$. coli also possessed a form of $\beta$-lactamase

229 and $12 \%$ possessed multiple $\beta$-lactamase genes. The extended spectrum $\beta$ -

230 lactamase, CTX-M was found significantly more frequently in monomicrobial

231 culture isolates than in isolates from polymicrobial infections $(P=0.014)$. To

232 determine phenotypic expression of extended spectrum resistance against $\beta$ -

233 lactam antibiotics the double-disc method was used to screen all $173 \beta$ - 
234 lactamase PCR positive strains. Sixteen strains (9\%) exhibited phenotypic ESBL

235 activity (12 E. coli, 3 Pseudomonas and 1 Enterobacter), most of which was in

236 strains that possessed multiple $\square$-lactamase genes, making it impossible to

237 identify the $\beta$-lactamase gene responsible for the extended spectrum resistance.

238 Detection of MRSA and PVL-producing S. aureus in polymicrobial UTI

239 samples

240 PCR detection of the mecA gene, confirmed $10(45 \%)$ of the urinary S. aureus

241 isolates to be MRSA. One of these was isolated from a monomicrobial culture

$242 \mathrm{UTI}$, and the remaining 9 confirmed MRSA were isolated from polymicrobial UTI

243 samples. Phenotypic resistance tests confirmed all PCR MRSA strains to be

244 resistant to cefoxitin, which is an indicator of methicillin resistance. The lukF

245 gene, which encodes PVL production was detected in 2 methicillin sensitive $S$.

246 aureus (MSSA) strains, which were both isolated from polymicrobial UTI

247 samples. No vancomycin resistance genes were found in any of the E. faecalis

248 isolates, but vanA was found in one $E$. faecium isolate. This isolate did not

249 however show any phenotypic resistance to vancomycin.

250 Bacteria isolated from polymicrobial UTI samples exhibit increased

251 pathogenic potential in in vitro cell invasion assays.

252 The ability to invade host epithelial cells is a critical factor in UTI. Therefore all $E$.

253 coli, E. faecalis, P. aeruginosa, P. mirabilis and $S$. aureus strains were

254 investigated to determine their ability to invade a human uroepithelial cell line.

255 T24 human bladder cells were infected with a bacterial culture for 3 hours, after

256 which external bacteria were killed by the addition of gentamicin and internalised

257 bacteria were enumerated. In the case of the $E$. coli assays, strains were 
258 designated as highly invasive if they showed a 10-fold increase in invasiveness

259 compared to that of the invasive reference strain, E. coli CFT073, which exhibited 260 variation of less than 1 log across all assays performed (less than 10 fold).

261 Overall 52 strains (34.7\%) were seen to exhibit the highly invasive phenotype 262 and 21 strains (14\%) exhibited a low invasive phenotype, more than a 10 fold 263 reduction in invasion compared to that of CFT073. E. coli isolates from mixed 264 culture samples exhibited increases in invasion as great as 1000 fold higher than 265 that observed in the invasive type strain CFT073. No invasive capacity was 266 observed in five strains, of which three were isolated from monomicrobial culture 267 samples where they were reported as the infectious agent. Overall $45 \%$ of the 268 polymicrobial E. coli strains assayed invaded to a similar level (less than a ten269 fold increase or decrease) as that of CFT073 (figure 3). This is not significantly 270 different to the monomicrobial culture isolates, $62 \%$ of which showed similar 271 invasion capability to CFT073. In contrast $44 \%$ of polymicrobial E. coli strains 272 were highly invasive, whereas no monomicrobial culture isolates invaded to a 273 greater level than CFT073 and $11 \%$ of polymicrobial isolates were classified as 274 'low invasive' compared to $38 \%$ of monomicrobial culture isolates. This suggests 275 that $E$. coli isolated from polymicrobial UTI samples may be significantly more 276 invasive $\left(P<0.001, X^{2}\right.$ distribution of strains with high, normal, and low invasive 277 capacity across polymicrobial and monomicrobial populations) in an in vitro 278 uroepithelial cell infection model, than E. coli isolated as monomicrobial cultures 279 from UTI samples. No association could be found between invasiveness and 280 patient gender, previous history of UTI, catheterisation status, health status of 281 patient, antibiotic resistance profile or BOX-PCR group. 
282 Invasion assays were also performed on all E. faecalis, $P$. mirabilis, $P$.

283 aeruginosa and S. aureus isolates. A wide range in invasion capability was noted

284 in E. faecalis isolates, ranging from $10^{6} \mathrm{cfu} / \mathrm{ml}$ to $10^{2} \mathrm{cfu} / \mathrm{ml}$ bacteria recovered

285 from invasion assays. P. mirabilis, $P$. aeruginosa and $S$. aureus showed a similar

286 trend with a 3-log range in invasion. Similarly the increased/decreased invasion

287 levels are not attributable to any patient characteristic, antibiotic resistance profile

288 or BOX-PCR group.

290 Discussion

291 Urinary tract infection rates in elderly people in the community can be as high as

$29210 \%$ at any one time, and this figure can rise to $30 \%$ of hospitalised patients.

293 Bacteraemic UTI in elderly patients can result in sepsis and death (Cove-Smith \&

294 Almond, 2007). A clinical microbiology laboratory will not routinely pursue or

295 report organisms present in mixed culture from urine samples unless there is a 296 significant count of a predominant organism. As up to $33 \%$ of samples can be 297 polymicrobial a large proportion of infections from the elderly population may go 298 untreated or indeed be treated with inappropriate antibiotics. This will only serve 299 to encourage the development of antibiotic resistance in urinary pathogens.

300 The predominant organism in polymicrobial UTI samples was E. coli, which 301 concurs with the general consensus among previously published data concerning 302 monomicrobial culture UTI (Farajnia et al., 2008, Tal et al., 2005, Johnson, 1991,

303 \& Brzuszkiewicz et al., 2006). E. faecalis was the second most commonly 304 isolated organism, and was significantly more prevalent in polymicrobial cultures 305 than monomicrobial cultures $(\mathrm{P}<0.001)$. E. faecalis and $E$. coli were found 
306 together in $36 \%$ of cultures and co-infection by these two pathogens may pose

307 important questions for the antibiotic treatment of polymicrobial UTI as

308 Enterococcus is known to be intrinsically resistant to many antibiotics, including

309 several first choice antibiotics for the treatment of UTI. The presence of both this

310 organism and uropathogenic $E$. coli in an infection may not only create difficulties

311 in devising an antibiotic treatment regimen but also recent studies have

312 suggested E. faecalis may exacerbate the pathogenicity of E. coli (Lavigne et al.,

313 2008, \& Montravers et al., 1997).

314 Prescription of ineffective antibacterial agents can increase selection pressure for

315 antibiotic resistant agents within an infection. Organisms present in polymicrobial

316 UTI cultures, that would not routinely be investigated, possessed antibiotic

317 resistance to front line antibiotics such as trimethoprim, ciprofloxacin and

318 amoxicillin. More importantly the percentage of mixed culture sample E. coli

319 isolates exhibiting resistance to trimethoprim and ciprofloxacin was higher than

320 that observed in monomicrobial culture sample isolates, and also higher than the

321 levels observed routinely in clinical isolates. Another issue raising concerns for

322 patient health is the presence of MRSA and PVL-producing MSSA in

323 polymicrobial UTI samples, which would not be detected under the current

324 guidelines for UTI diagnosis. The presence of MRSA in an infection limits the

325 choice of antibiotics available for treatment, and the cytotoxin PVL attacks white

326 blood cells and can cause severe tissue necrosis (Holmes et al., 2005). Other

327 specific antibiotic resistance traits such as extended-spectrum $\beta$-lactamases

328 were also detected, by PCR for known $\beta$-lactamase genes, although only a small

329 proportion showed phenotypic activity. This discrepancy re-emphasises the 
330 importance of screening clinical isolates for ESBL gene carriage as opposed to

331 phenotypic tests (Livermore \& Hawkey, 2005, Tofteland et al., 2007, \& Xu et al.,

332 2005). Interestingly, the current increase in isolation of $E$. coli 025b-ST131 CTX-

$333 \mathrm{M}^{\mathrm{R}}$ from clinical samples (Lau et al., 2008, Nicolas-Chanoine et al., 2008, \&

334 Vincent et al., 2010) does not appear to be reflected in this study, with only $11 \%$

335 of $E$. coli containing the blaстх-м gene. The full genetic diversity and lineage of the

336 EXPEC strains isolated in this study is the current focus of intensive research.

337 It has been suggested that the critical step in UTI initiation is the attachment to

338 and invasion of the superficial bladder epithelium, especially in the case of $E$. coli

339 (Anderson et al., 2003, \& Mulvey et al., 2001). By attaching to bladder epithelial

340 cells $E$. coli are able to establish reservoirs known as intra-cellular bacterial

341 communities, from which the invading bacteria receive some level of protection

342 against the host immune system and also initiate recurrent infections. Therefore

343 invasive bacteria are considered more proficient in instigating an infection.

344 Significant differences were identified in the invasive capabilities of

345 monomicrobial culture and polymicrobial culture isolates (figure 3). E. coli isolates

346 of polymicrobial culture origin were significantly more invasive when compared to

347 the invasion of the type strain, E. coli CFT073, than strains isolated from

348 monomicrobial culture samples. The majority of isolates from monomicrobial

349 infections were less invasive than E. coli CFT073 and none exhibited a highly

350 invasive phenotype. The polymicrobial isolates that possessed increased

351 invasive capacity did not belong exclusively to any specific patient group and

352 were not shown to be associated with patient gender, catheterisation status,

353 previous history of UTI and underlying medical issues, antibiotic resistance profile 
354 or BOX-PCR group. This indicates the potential existence of a heterogeneous

355 group of highly invasive E. coli within polymicrobial UTI in the elderly, which

356 would not be diagnosed or treated due to limitations in the current diagnostic

357 standard procedure. A further study including larger numbers of ExPEC isolates

358 from monomicrobial infections is required to rule out any such associations, and

359 indeed to confirm the significance of the increased invasive phenotype

360 exclusively observed in the polymicrobial isolates from this study. This is

361 currently under investigation in several hospital labs examining equal numbers of

362 polymicrobial and monomicrobial isolates, including an examination of the clinical

363 nature of the infections, their association with complicated or uncomplicated UTI,

364 and the genotypic and phenotypic differences associated with the hyper-invasive

365 phenotype.

366 The frequent co-isolation of E. coli and E. faecalis from the clinical UTI samples

367 raises questions as to the possible contribution of Enterococci to the increased

368 invasive phenotype expressed by the ExPEC strains isolated in this study. There

369 have been reports of Enterococci exacerbating the pathogenicity of other

370 organisms including ExPEC in both $C$. elegans and rat models of infection

371 (Lavigne et al., 2008, \& Montravers et al., 1997), and the promiscuous nature of

372 Enterococci with regards to gene transfer is well known. The possibility that

373 Enterococci can alter the genotype and/or phenotype of ExPEC during co-

374 infection of bladder epithelial cells is currently the subject of further investigation.

375 Polymicrobial urinary tract infections may pose a heightened threat to the health

376 and well being of the elderly population. This study found that the organisms

377 present in polymicrobial UTI possess traits such as antibiotic resistance akin to 
378 that of their monomicrobial culture counterparts, and potentially with increased

379 resistance to ciprofloxacin and trimethoprim which common front line antibiotics

380 used for UTI treatment. Due to the complexities involved in the diagnosis and

381 treatment in these infections many patients may receive inadequate antibiotic

382 treatment or indeed a lack of treatment altogether. More worryingly for patient

383 health, the majority of organisms isolated from polymicrobial cultures also

384 exhibited increased human pathogenic potential as evidenced by in vitro cell

385 infection assays. The diagnostic standard procedure for UTI should be

386 reconsidered in light of the data presented here.

387 Acknowledgements:

388 We would like to thank Prof David Gally, University of Edinburgh for kindly 389 supplying E. coli CFT073. We would also like to thank Graham White, Caroline

390 Bland, Adele Wasson, Richard Shaw, and staff from the Nottingham University

391 Hospitals clinical microbiology laboratory for their invaluable assistance. Also

392 thanks to Mark Collery from the University of Nottingham and Katarzyna Lys-

393 Slimak from Nottingham Trent University for VRE and MRSA PCR. 
396 1. Anderson, G. G., Palermo, J. J., Schilling, J. D., Roth, R., Heuser, J. \& Hultgren, S. J. (2003). Intracellular bacterial biofilm-like pods in urinary tract infections. Science 301, 105-107.

2. Andrews, J. (2009). BSAC standardized disc susceptibility testing method (version 8). Journal of Antimicrobial Chemotherapy 64, 454-489.

3. Blango, M. G. Mulvey, M. A. 2010. Persistence of uropathogenic Escherichia coli in the face of multiple antibiotics. Antimicrob Agents Chemother. doi:10.1128/AAC.00014-10

4. Brzuszkiewicz, E., Bruggemann, H., Liesegang, H., Emmerth, M., Oelschlaeger, T., Nagy, G., Albermann, K., Wagner, C., Buchrieser, C., Emody, L., Gottschalk, G., Hacker, J. \& Dobrindt, U. (2006). How to become a uropathogen: Comparative genomic analysis of extraintestinal pathogenic Escherichia coli strains. PNAS 103, 12879-12884.

5. Cove-Smith, A. \& Almond, M. (2007). Management of urinary tract infections in the elderly. Trends in Urology, Gynaecology \& Sexual Health $12,31-34$

6. Fang, H., Ataker, F., Hedin, G. \& Dornbusch, K. (2008). Molecular epidemiology of extended-spectrum $\beta$-lactamases among Escherichia coli isolates collected in a Swedish hospital and its associated health care facilities from 2001 to 2006. Journal of Clinical Microbiology 46, 707-712.

7. Farajnia, S., Alikhani, M. Y., Ghotaslou, R., Naghili, B. \& Nakhlband, A. (2008). Causative agents and antimicrobial susceptibilities of urinary tract infections in the northwest of Iran. International Journal of Infectious 
Diseases 13, 140-144.

420 8. Holmes, A., Ganner, M., McGuane, S., Pitt, T. L., Cookson, B. D. \& Kearns, A. M. (2005). Staphylococcus aureus isolates carrying PantonValentine Leucocidin genes in England and Wales: frequency, characterization, and association with clinical disease. Journal of Clinical

9. Javed, M. A., Grant, A. J., Bagnall, M. C., Maskell, D. J., Newell, D. G.

\& Manning, G. (2009). Transposon mutagenesis in a hyper-invasive clinical isolate of Campylobacter jejuni reveals a number of genes with potential roles in invasion. Microbiology 156(4), 1134-1143. genotypes of vancomycin-resistant Enterococci in nosocomial surveillance specimens by PCR. Journal of Clinical Microbiology 37, 2090-2092.

11. Johnson, J. R. 1991. Virulence factors in Escherichia coli urinary tract infection. Clin Microbiol Rev 4:80-128. subsequence conservation of interspersed repetitive Streptococcus pneumoniae BOX elements in diverse bacteria. Genome Research 5, 408418.

13. Kumazawa, J., \& Matsumoto, T. (1997). Complicated Urinary Tract Infections. In Urinary Tract Infections: Molecular Pathogenesis and Clinical Management, pp. 19-26. Edited by T. Bergan. Basel, Switzerland: Karger Publishers.

\section{Lau, S. H., Kaufmann, M. E., Livermore, D. M., Woodford, N.,}


Willshaw, G. A., Cheasty, T., Stamper, K., Reddy, S., Cheesbrough, J., coli strains A-E, with CTX-M-15 b-lactamase, all belong to the international O25:H4-ST131 clone. Journal of Antimicrobial Chemotherapy 62, 1241-1244.

15. Lavigne, J.-P., Nicolas-Chanoine, M.-H., Bourg, G., Moreau, J. \& Sotto, A. (2008). Virulent synergistic effect between Enterococcus faecalis and Escherichia coli assayed by using the Caenorhabditis elegans model. PLOS ONE 3, e3370.

16. Livermore, D. M. \& Hawkey, P. M. (2005). CTX-M: changing the face of ESBLs in the UK. Journal of Antimicrobial Chemotherapy 56, 451-454.

\section{McNally, A., Ragione, R. M. L., Best, A., Manning, G. \& Newell, D. G.} (2007). An aflagellate mutant Yersinia enterocolitica biotype 1A strain displays altered invasion of epithelial cells, persistence in macrophages, and cytokine secretion profiles in vitro. Microbiology 153, 1339-1349.

18. Montravers, P., Mohler, J., Julien, L. S. \& Carbon, C. (1997). Evidence of the proinflammatory role of Enterococcus faecalis in polymicrobial peritonitis in rats. Infection and Immunity 65, 144-149.

19. Mulvey, M. A., Schilling, J. D. \& Hultgren, S. J. (2001). Establishment of a persistent Escherichia coli reservoir during the acute phase of a bladder infection. Infection and Immunity 69, 4572-4579.

20. Nicolas-Chanoine, M.-H., Blanco, J., Leflon-Guibout, V., Demarty, R., Alonso, M. P., Canic, M. M., Park, Y.-J., Lavigne, J.-P., Pitout, J. \& Johnson, J. R. (2008). Intercontinental emergence of Escherichia coli 
clone O25:H4-ST131 producing CTX-M-15. Journal of Antimicrobial Chemotherapy 61, 273-281.

21. Nicolle, L. E. (2001a). Urinary tract infections in long-term-care facilities. Infection Control and Hospital Epidemiology 22, 167-175.

22. Nicolle, L. E. (2001b). Urinary tract pathogens in complicated infection and in elderly individuals. The Journal of Infectious Diseases 183, S5-S8. Cookson, B. \& L.Taylor (2001). The rate and cost of hospital-acquired infections occurring in patients admitted to selected specialties of a district general hospital in England and the national burden imposed. Journal of Hospital Infection 47, 198-209.

24. Proudy, I., Bougle, D., Leclercq, R. \& Vergnaud, M. (2008). Tracing of Enterobacter sakazakii isolates in infant milk formula processing by BOXPCR genotyping. Journal of Applied Microbiology 105, 550-558.

First Report of Infection with community-acquired Methicillin-Resistant Staphylococcus aureus in South America. Journal of Clinical Microbiology 43, 1985-1988.

26. Tal, S., Guller, V., Levi, S., Bardenstein, R., Berger, D., Gurevich, I. \& Gurevich, A. (2005). Profile and prognosis of febrile elderly patients with bacteremic urinary tract infection. Journal of Infection 50, 296-305.

27. Thomas, L. C., Gidding, H. F., Ginn, A. N., Olma, T. \& Iredell, J. (2007). Development of a real-time Staphylococcus aureus and MRSA (SAM-) 
PCR for routine blood culture. Journal of Microbiological Methods 68, 296302.

28. Tofteland, S., Haldorsen, B., Dahl, K. H., Simonsen, G. S., Steinbakk, M., Walsh, T. R., Sundsfjord, A. \& Group, T. N. E. S. (2007). Effects of phenotype and genotype on methods for detection of Extended-SpectrumB-Lactamase-producing clinical isolates of Escherichia coli and Klebsiella pneumoniae in Norway. Journal of Clinical Microbiology 45, 199-205. 29. Vincent, C., Boerlin, P., Daignault, D., Dozois, C. M., Dutil, L., Galanakis, C., Reid-Smith, R. J., Tellier, P.-P., Tellis, P. A., Ziebell, K. \& Manges, A. R. (2010). Food reservoir for Escherichia coli causing urinary tract infections. Emerging Infectious Diseases 16, 88-95.

30.Xu, L., Ensor, V., Gossain, S., Nye, K. \& Hawkey, P. (2005). Rapid and simple detection of blaCTX-M genes by multiplex PCR assay. Journal of Medical Microbiology 54, 1183-1187.

31. Yang, H.-H., Vinopal, R. T., Grasso, D. \& Smets, B. F. (2004). High diversity among environmental Escherichia coli isolates from a bovine feedlot. Applied and Environmental Microbiology 70, 1528-1536.

32.Zhanel, G. G., Hisanaga, T. L., Laing, N. M., DeCorby, M. R., Nichol, K. A., Palatnick, L. P., Johnson, J., Noreddin, A., Harding, G. K. M., Nicolle, L. E., Group, t. N. \& Hobana, D. J. (2005). Antibiotic resistance in outpatient urinary isolates: final results from the North American Urinary Tract Infection Collaborative Alliance (NAUTICA). International Journal of Antimicrobial Agents 26, 380-388. 


\begin{tabular}{|c|c|c|c|c|c|c|c|c|c|c|}
\hline \multirow{2}{*}{$\begin{array}{c}\text { Antibiotic } \\
\text { concentration } \\
(\mu \mathrm{g} / \mathrm{ml})\end{array}$} & \multicolumn{2}{|c|}{ E. coli $(\%)$} & \multicolumn{2}{|c|}{ E. faecalis (\%) } & \multicolumn{2}{|c|}{ P. mirabilis (\%) } & \multicolumn{2}{|c|}{ S. aureus (\%) } & \multicolumn{2}{|c|}{$\begin{array}{c}\text { P. aeruginosa } \\
(\%)\end{array}$} \\
\hline & $\begin{array}{l}\text { Poly } \\
(n=129)\end{array}$ & $\begin{array}{l}\text { Mono } \\
(n=21)\end{array}$ & $\begin{array}{c}(\mathrm{n}=110 \\
)\end{array}$ & $\begin{array}{l}\text { Mono } \\
(n=4)\end{array}$ & $\begin{array}{l}\text { Poly } \\
(n=56)\end{array}$ & $\begin{array}{l}\text { Mono } \\
(n=1)\end{array}$ & $\begin{array}{l}\text { Poly } \\
(n=18)\end{array}$ & $\begin{array}{c}0 \\
(n=4)\end{array}$ & $\begin{array}{l}\text { Poly } \\
(n=46)\end{array}$ & $\begin{array}{l}\text { Mono } \\
(n=5)\end{array}$ \\
\hline Gentamicin (2) & 12.4 & 4.76 & - & - & 5.3 & 0.0 & 4.5 & 0 & - & - \\
\hline Cefotaxime (1) & 17.8 & 14.29 & - & - & 33.9 & 100.0 & - & - & - & - \\
\hline Ceftazidime (1) & 18.6 & 9.52 & - & - & 35.7 & 100.0 & - & - & - & - \\
\hline Meropenem (2) & 0.0 & 0.00 & 15.5 & 0.0 & 0.0 & 0.0 & - & - & - & - \\
\hline $\begin{array}{l}\text { Piperacillin- } \\
\text { Tazobactam (16) }\end{array}$ & 6.2 & 4.76 & 4.5 & 25.0 & 23.2 & 100.0 & - & - & - & - \\
\hline $\begin{array}{l}\text { Co-amoxiclav } \\
\text { (32) }\end{array}$ & 5.4 & 0.00 & 2.7 & 0.0 & 16.1 & 100.0 & 0.0 & 0.0 & - & - \\
\hline Trimethoprim (2) & 44.2 & 28.57 & - & - & 89.0 & 0.0 & 22.2 & 0.0 & - & - \\
\hline Ciprofloxacin (4) & 23.3 & 9.52 & 28.2 & 0.0 & 0.0 & 0.0 & 55.5 & 50.0 & - & - \\
\hline Cephradine (32) & 28.7 & 19.05 & - & - & 55.3 & 100.0 & 50.0 & 25.0 & - & - \\
\hline $\begin{array}{l}\text { Nitrofurantoin } \\
\text { (32) }\end{array}$ & 17.1 & 9.52 & 10.9 & 0.0 & & & 11.0 & 0.0 & - & - \\
\hline Amoxicillin (32) & 45.0 & 42.86 & 7.3 & 0.0 & 37.5 & 100.0 & 66.6 & 75.0 & - & - \\
\hline Gentamicin (10) & - & - & - & - & - & - & - & - & 2.17 & 0.0 \\
\hline $\begin{array}{l}\text { Piperacillin- } \\
\text { tazobactam (85) }\end{array}$ & - & - & - & - & - & - & - & - & 0.0 & 0.0 \\
\hline Ceftazidime (30) & - & - & - & - & - & - & - & - & 2.17 & 00.0 \\
\hline Meropenem (10) & - & - & - & - & - & - & - & - & 2.17 & 0.0 \\
\hline Ciprofloxacin (1) & - & - & - & - & - & - & - & - & 4.35 & 00.0 \\
\hline
\end{tabular}

\section{Table 1. Prevalence of antibiotic resistance in UTI isolates}


NOTE '-' indicates that particular species/antibiotic combination was not tested.

The term 'poly' refers to strains of polymicrobial infection origin, whilst the term 'mono' refers to strains of monomicrobial infection origin. 
Table 2. $\beta$-lactamase gene carriage in UTI isolates as determined by PCR

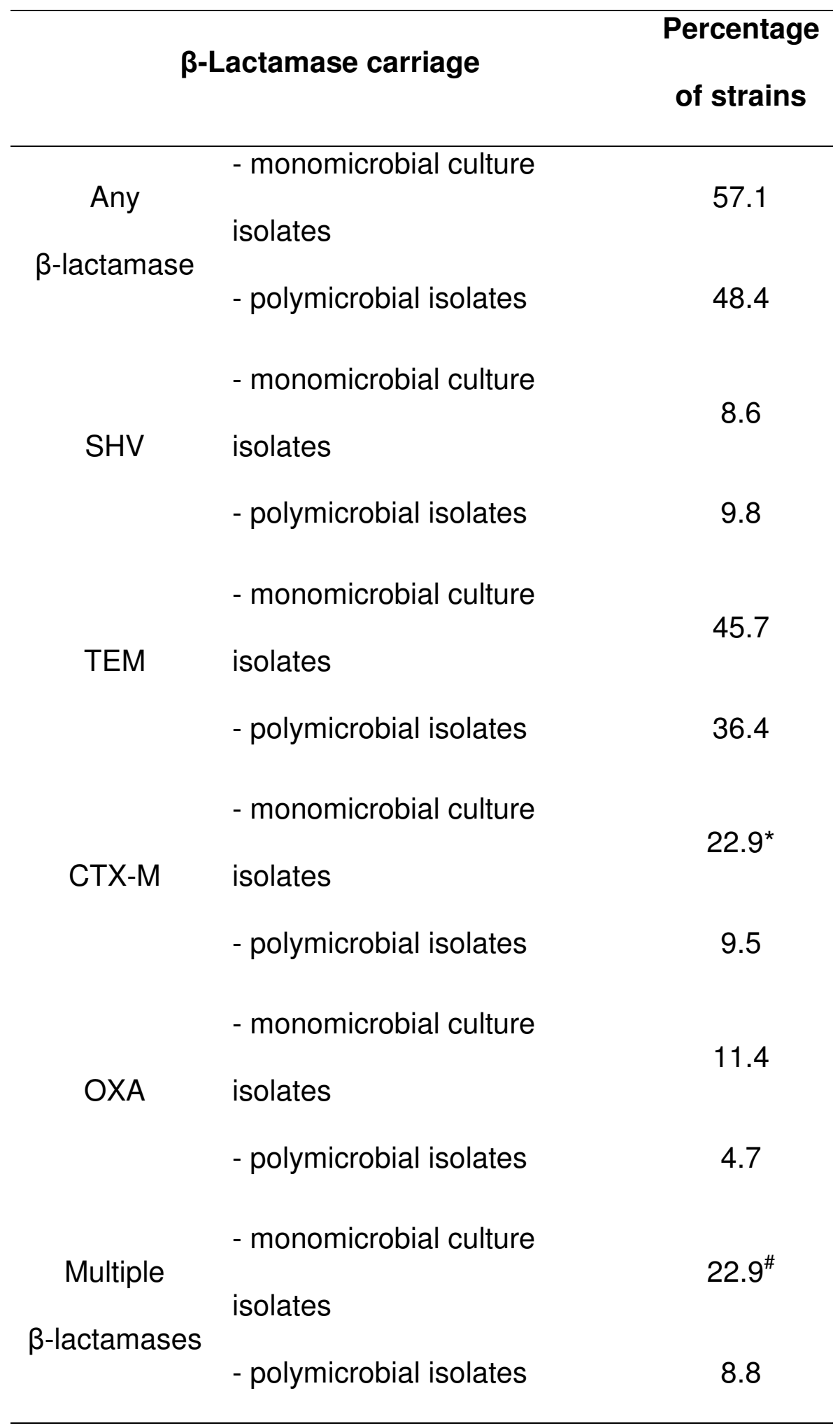


Strains tested were all E.. coli, Pseudomonas spp, Proteus spp, Citrobacter spp, Enterobacter spp and Klebsiella spp isolated in this study. ${ }^{*} \mathrm{P}=0.016, \# \mathrm{P}=0.01, \mathrm{X}$ ${ }^{2}$ test. 
Figure 1.The relative prevalence of each of the main species associated with UTI isolated from the different types of sample collected in the study. Prevalence is presented as the percentage of samples collected which contain the given species.

* Indicates cultures taken from polymicrobial infections

Figure 2. BOX-PCR gel of E. coli strains isolated from UTI samples Lanes marked * contain the BOX-PCR profile for Escherichia coli CFT073, lanes marked M contain molecular weight markers. Remaining lanes show a selection of BOX profiles obtained for $E$. coli isolates.

Figure 3. Invasive capabilities of UTI E. coli isolates compared to E. coli CFT073. CFT073 invasion is designated as 1.00 . Polymicrobial sample isolates are statistically more invasive than monomicrobial culture sample isolates, $P<0.001, x^{2}$ test. 